ARTICLE

DOI: $10.1038 / s 41467-017-00034-4$

\title{
Cellulose-based magnetoelectric composites
}

\author{
Yan Zong ${ }^{1}$, Tian Zheng ${ }^{1}$, Pedro Martins ${ }^{2}$, S. Lanceros-Mendez ${ }^{2,3,4}$, Zhilian Yue ${ }^{1} \&$ Michael J. Higgins ${ }^{1}$
}

Since the first magnetoelectric polymer composites were fabricated more than a decade ago, there has been a reluctance to use piezoelectric polymers other than poly (vinylidene fluoride) and its copolymers due to their well-defined piezoelectric mechanism and high piezoelectric coefficients that lead to superior magnetoelectric coefficients of $>1 \mathrm{~V} \mathrm{~cm}^{-1} \mathrm{Oe}^{-1}$. This is the current situation despite the potential for other piezoelectric polymers, such as natural biopolymers, to bring unique, added-value properties and functions to magnetoelectric composite devices. Here we demonstrate a cellulose-based magnetoelectric laminate composite that produces considerable magnetoelectric coefficients of $\approx 1.5 \mathrm{~V} \mathrm{~cm}{ }^{-1} \mathrm{Oe}^{-1}$, comprising a Fano resonance that is ubiquitous in the field of physics, such as photonics, though never experimentally observed in magnetoelectric composites. The work successfully demonstrates the concept of exploring new advances in using biopolymers in magnetoelectric composites, particularly cellulose, which is increasingly employed as a renewable, low-cost, easily processable and degradable material.

\footnotetext{
${ }^{1}$ ARC Centre for Electromaterials Science (ACES), Intelligent Polymer Research Institute/AllM Faculty, Innovation Campus, Squires Way, University of Wollongong, Wollongong, NSW 2522, Australia. ${ }^{2}$ Centro/Departamento de Física, Universidade do Minho, Braga 4710-057, Portugal. ${ }^{3}$ BCMaterials, Basque Center for Materials, Applications and Nanostructures, Parque Tecnologico de Bizkaia, Derio 48160, Spain. ${ }^{4}$ IKERBASQUE, Basque Foundation for Science, Bilbao 48013, Spain. Correspondence and requests for materials should be addressed to M.J.H. (email: mhiggins@uow.edu.au)
} 
M agnetoelectric (ME) materials undergo dielectric (magnetic) polarization in an external magnetic (electric) field ${ }^{1,2}$. Compared to rare single phase ME crystals (e.g., $\left.\mathrm{Cr}_{2} \mathrm{O}_{3}\right)^{3}$, composites with $\mathrm{ME}$ properties are attractive for their ease of processing and, importantly, superior ME response at room temperature $e^{4,5}$ to enable practical devices such as sensors for ultralow magnetic field detection ${ }^{6}$. ME composites consist of both piezoelectric and magnetostrictive components, and the ME effect of the composite is not the natural property per se, but is actually the result of tensor properties $^{7}$. That is, when an external magnetic field is applied to the composite, the magnetic component changes its shape magnetostrictively to induce strain on the piezoelectric component, causing dielectric polarization. This uniquely indirect two-phase strain coupling provides the flexibility of optimizing both the piezoelectric phase, magnetostrictive phase, and their interface to enhance the ME response ${ }^{8}$.

Generally, the use of magnetostrictive materials with high magnetic permeability and low field saturation, such as Metglas 9,10 , provides the possibility of inducing fast mechanical deformation under relatively weak magnetic fields. Combining Metglas with the highest piezoresponsive polymers, such as poly(vinylidene fluoride) $(\mathrm{PVDF})^{11}$, gives rise to significant strain transfer and the highest ME voltage outputs. As such, PVDF and its copolymers have been exclusively studied since the first ME polymer composite consisting of PVDF was demonstrated in 2002 and expected developments in exploring other types of piezoelectric polymers have not been forthcoming. This has led to the emergence of a central dogma where PVDF is viewed as the "material of merit", despite the enormous potential for other polymers to bring significant added-value properties and function to ME composite devices. Actually in nature, there are numerous biomolecules possessing piezoelectricity and the practical applications arising from them has attracted a lot of research interest $^{12,13}$. Exploring advances in harnessing the unique structures and properties of naturally occurring piezoelectric biopolymers will transpose the aforementioned central dogma and broaden the application base for ME composites. In particular, cellulose is the most substantial organic substance in nature $^{14}$ and the origin of its piezoelectric response, discovered early in wood ${ }^{15}$, comes from inherent crystallinity ${ }^{16}$ (Fig. 1a). As a very cheap and renewable material, it is developing rapidly as a supporting substrate for flexible and transparent electronics ${ }^{17}, 18$, and recently fabricated as piezoelectric paper for actuation, energy harvesting, and acoustic applications ${ }^{19}$.

Here we first demonstrate the fabrication of ME composites based on the natural biopolymer, cellulose. In regenerated cellulose, the degree of crystallinity is usually at a low level and improving the alignment of cellulose fibrils, e.g., either through stretching or heat, is an effective method to enhance piezoelectricity, as the ordered structure (Fig. 1b) is preferred for crystalline generation $^{19,20}$. To fabricate the cellulose-based ME composites, we prepare laminate (bilayer) structures comprising Metglas and cellulose films, enabling two-phase strain coupling for an enhanced ME response, that is superior to previously reported composite structures ${ }^{21}$. Cellulose films are obtained from regular solution processing methods and the pristine wet film is aired at room temperature or alternatively hot pressed $\left(60^{\circ} \mathrm{C}\right)$ to assess the effect of heat treatment on the cellulose crystallinity and overall ME response (see 'Methods' section).
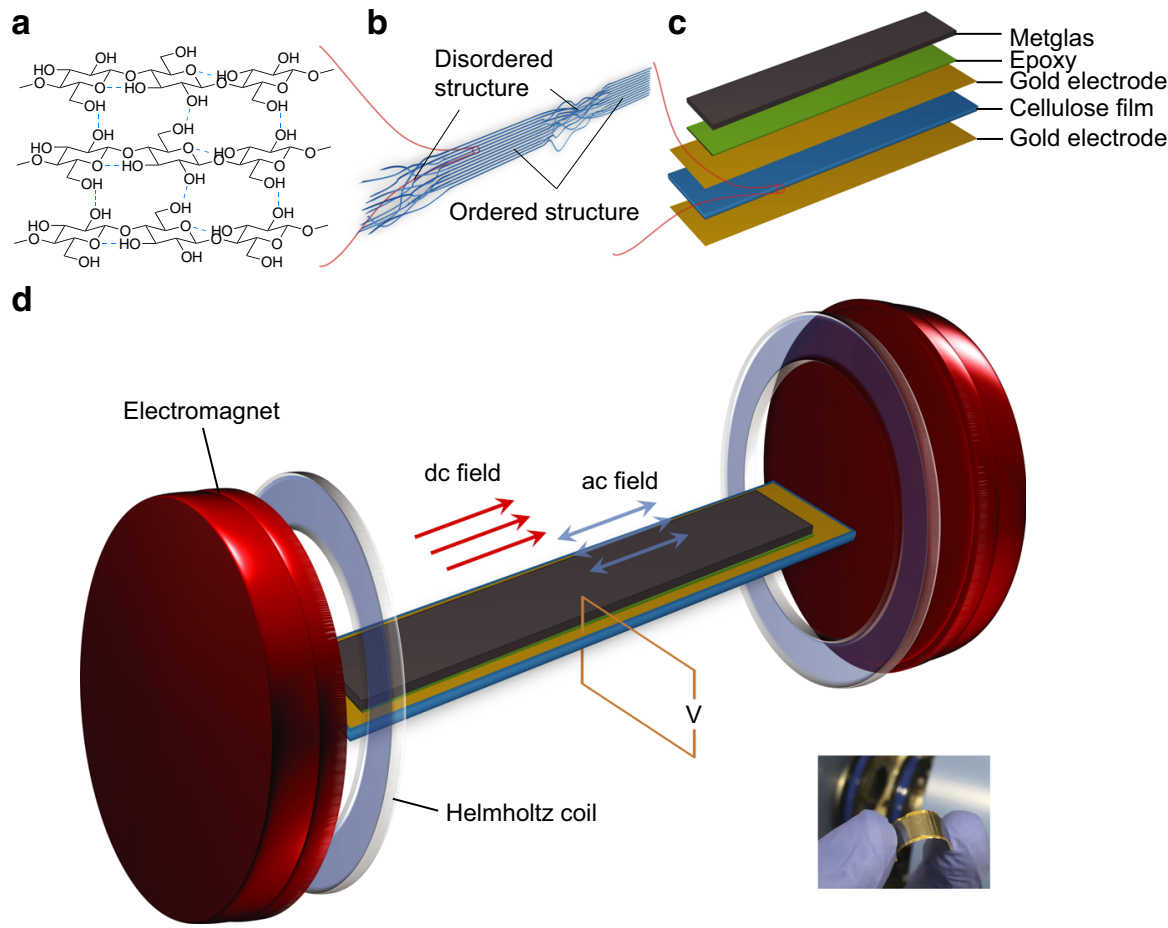

Fig. 1 Cellulose ME laminate and the experimental set-up of ME effect measurement. a Scheme of cellulose crystal II, the most common crystalline type in regenerated cellulose materials. The saccharide unit provides dipolar segments along the aligned fibril thus rendering the piezoelectric nature. $\mathbf{b}$ Illustration of cellulose fibril alignment at the cross-section of cellulose film. Part of the ordered structure provides crystalline properties. c Schematic of cellulosebased ME laminate structure. The thickness is measured as $19 \pm 2 \mu \mathrm{m}$ for hot-press film and $27 \pm 3 \mu \mathrm{m}$ for the control film by using a micrometer caliper. The cellulose films are first sputter coated with $50 \mathrm{~nm}$ thick gold electrodes on both sides and then glued with Metglas 2605 SA1, of which the thickness is $25 \mu \mathrm{m}$. To ensure even distribution, the epoxy is preheated to $60^{\circ} \mathrm{C}$ to improve the liquidity. d Schematic view of the bulk system for ME voltage measurement. The output voltage is collected from the interface gold electrodes and monitored as root mean square values using a lock-in amplifier. The inset below shows the picture of the final cellulose ME laminate used in the measurements 

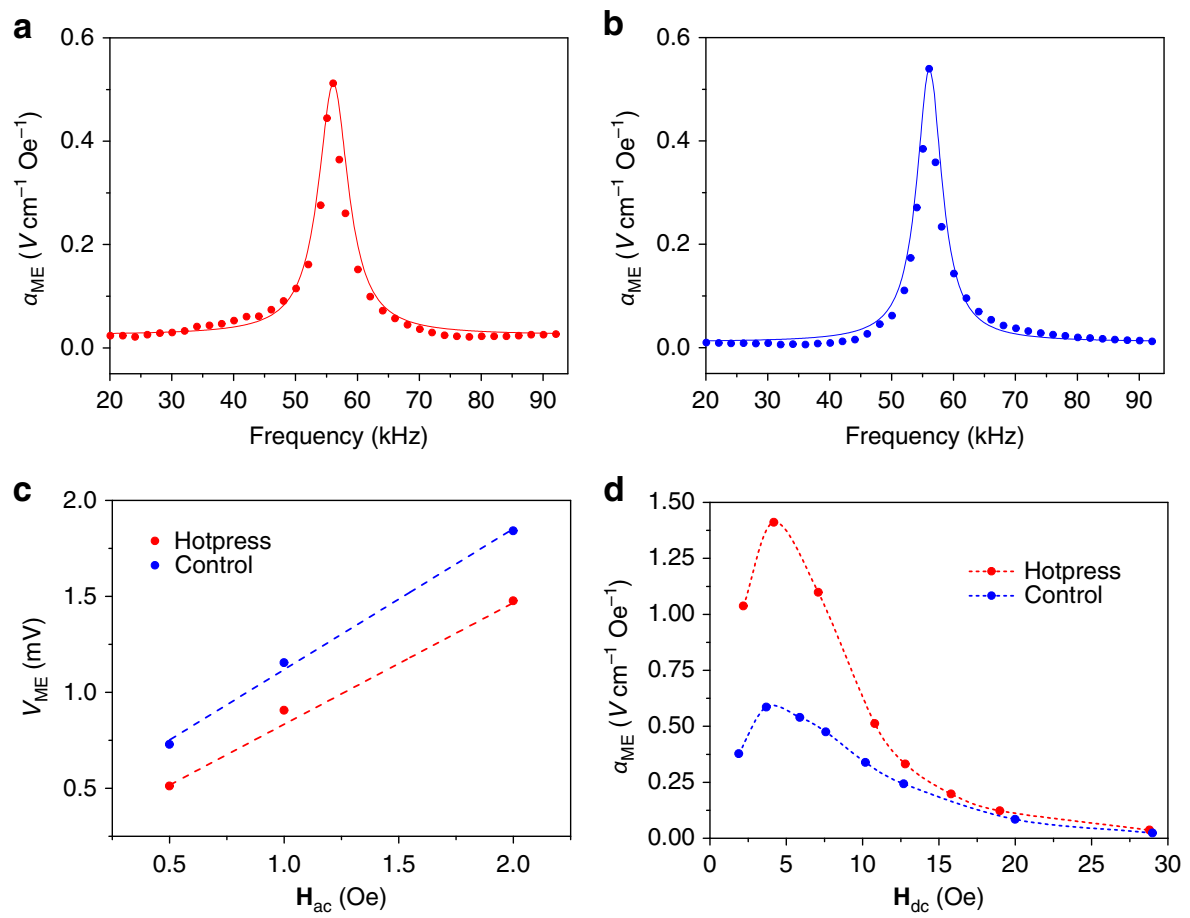

Fig. 2 ME effect of cellulose-Metglas laminates. a, b ME voltage coefficient as a function of $\mathbf{H}_{\mathrm{ac}}$ frequency when $\mathbf{a} \mathbf{H}_{\mathrm{dc}}=10.8$ Oe for hot-press sample and $\mathbf{b}$ $\mathbf{H}_{\mathrm{dc}}=5.9$ Oe for the control sample. The experimental data points are fitted to a Lorentzian resonance model (the solid line) and the resonance peaks found at $56.1 \mathrm{kHz}$. c The resonant ME output voltage as a function of applied $\mathbf{H}_{\mathrm{dc}}$ strength. The ME laminates were induced by $\mathbf{H}_{\mathrm{dc}}=10.8 \mathrm{Oe}$ for hot-press sample and $\mathbf{H}_{\mathrm{dc}}=5.9$ Oe for the control sample at which a Lorentzian resonance profile has been observed. $\mathbf{d}$ Resonance enhanced $\alpha_{\mathrm{ME}}$ as a function of $\mathbf{H}_{\mathrm{dc}}$ for hot press (red dotted line) and control (blue dotted line). All data are obtained under $\mathbf{H}_{\mathrm{ac}}=0.5 \mathrm{Oe}$

ME laminates are assembled by combining a cellulose film, consisting of gold layers sputtered on both sides as inter-face electrodes, with a Metglas (the magnetostrictive phase) film by gluing the two components together using Devcon epoxy to enable strain coupling (Fig. 1c). The final laminate composite is tested using a dynamic method, involving the application of an alternating $\left(\mathbf{H}_{\mathrm{ac}}\right)$ magnetic field superimposed on a constant $\left(\mathbf{H}_{\mathrm{dc}}\right)$ magnetic field, to quantify the ME frequency response and output voltage (Fig. 1d).

\section{Results}

ME properties of cellulose/Metglas laminate composites. The ME voltage coefficient $\left(\alpha_{\mathrm{ME}}\right)$ is an important parameter that evaluates the ME effect of a material, and is defined as:

$$
\alpha_{\mathrm{ME}}=\frac{\mathrm{d} \mathbf{E}}{\mathrm{d} \mathbf{H}}
$$

where $\mathbf{E}$ and $\mathbf{H}$ represent the strength of the electrical and magnetic fields, respectively. $\alpha_{\mathrm{ME}}$ is calculated as the ME output voltage $(V)$ per unit of cellulose film thickness $(\mathrm{cm})$ and $\mathbf{H}_{\mathrm{ac}}$ strength (Oe). To measure the ME response, we first fix the $\mathbf{H}_{\mathrm{dc}}$ and $\mathbf{H}_{\mathrm{ac}}$ strength and $\alpha_{\mathrm{ME}}$ is recorded as the function of the $\mathbf{H}_{\mathrm{ac}}$ frequency. For both the air-dried and hot-pressed cellulose ME laminates, a significant increase in $\alpha_{\mathrm{ME}}$ is observed with a peak maximum at $\mathbf{H}_{\mathrm{ac}}$ frequency of $\sim 56.1 \mathrm{kHz}$ (Fig. 2a, b), indicating a resonance enhancement effect that is characteristic of an $\mathrm{ME}$ response in laminate structures ${ }^{21}$. In this case, once the $\mathbf{H}_{\mathbf{a c}}$ frequency coincides with the resonant frequency of the magnetostrictive structure (i.e., Metglas film), the mechanical strain is amplified, thus causing a significant enhancement in the $\alpha_{\mathrm{ME}}$. The resonant frequency $\left(f_{\mathrm{r}}\right)$ of the magnetostrictive layer, if oscillating with a free end, depends on its density $(\rho)$, Young's modulus $(E)$, and the length $(L)$ along the magnetic field ${ }^{22}$, and is given by:

$$
f_{\mathrm{r}}=\frac{1}{2 L} \times \sqrt{E / \rho}
$$

For Metglas, $E=100-110 \mathrm{GPa}$ and $\rho=7.18 \times 10^{3} \mathrm{~kg} \mathrm{~m}^{-3}$. In our case $L=36 \mathrm{~mm}$, thus the calculated theoretical $f_{\mathrm{r}}$ of $51.8-54.4$ $\mathrm{kHz}$ is very close to the experimental $\alpha_{\mathrm{ME}}$ resonance peak values in Fig. 2a, b. For Fig. 2a, b, ME responses are specifically shown for different applied $\mathbf{H}_{\mathrm{dc}}$ of 10.8 Oe and 5.9 Oe for the hot-pressed and air-dried samples, respectively, because these $\mathbf{H}_{\mathrm{dc}}$ produce a symmetrical Lorentzian resonance profile as opposed to a non-symmetrical profile, which is discussed later in Fig. 3. Displayed in Fig. 2c is the actual resonant output voltage $\left(V_{\mathrm{ME}}\right)$ under different $\mathbf{H}_{\mathrm{ac}}$ strength while the $\mathbf{H}_{\mathrm{dc}}$ strength is fixed at the respective values given in Fig. $2 \mathrm{a}, \mathrm{b}$. Under these $\mathbf{H}_{\mathrm{dc}}$ strengths, the $V_{\mathrm{ME}}$ of the air dried is slightly higher than the hot pressed, and the linear increase in both as a function of $\mathbf{H}_{\mathrm{ac}}$ further confirms the existence of an ME effect (Fig. 2c). Despite the airdried samples having higher $V_{\mathrm{ME}}$ for the symmetrical Lorentzian resonance condition, the hot-pressed samples gave significantly higher $\alpha_{\mathrm{ME}}$ at all other $\mathbf{H}_{\mathrm{dc}}$ strengths (Fig. 2d). More specifically, Fig. $2 \mathrm{~d}$ shows the resonance $\alpha_{\mathrm{ME}}$ as a function of $\mathbf{H}_{\mathrm{dc}}$ strength. The hot-press samples show a maximum $\alpha_{\mathrm{ME}}$ at $\mathbf{H}_{\mathrm{dc}}=4.2 \mathrm{Oe}$, as high as $1.41 \mathrm{~V} \mathrm{~cm}^{-1} \mathrm{Oe}^{-1}$, which is importantly considered to be in the practically useful range ${ }^{11}$. Comparatively, the air-dried sample shows a similar $\mathbf{H}_{\mathrm{dc}}$-dependent behavior, with significantly less maximum $\alpha_{\mathrm{ME}}$ of $0.59 \mathrm{~V} \mathrm{~cm}^{-1} \mathrm{Oe}^{-1}$ occurring at $\mathbf{H}_{\mathrm{dc}}=3.9$ Oe. Furthermore, it is expected that the maximum ME output voltage can be further enhanced by increasing $\mathbf{H}_{\mathrm{ac}}$, as given by the linear relationship (i.e., $1.48 \mathrm{mV}$ for the hot-pressed sample when $\mathbf{H}_{\mathrm{ac}}=2.0 \mathrm{Oe}$ ) in Fig. $2 \mathrm{c}^{23,24}$. 

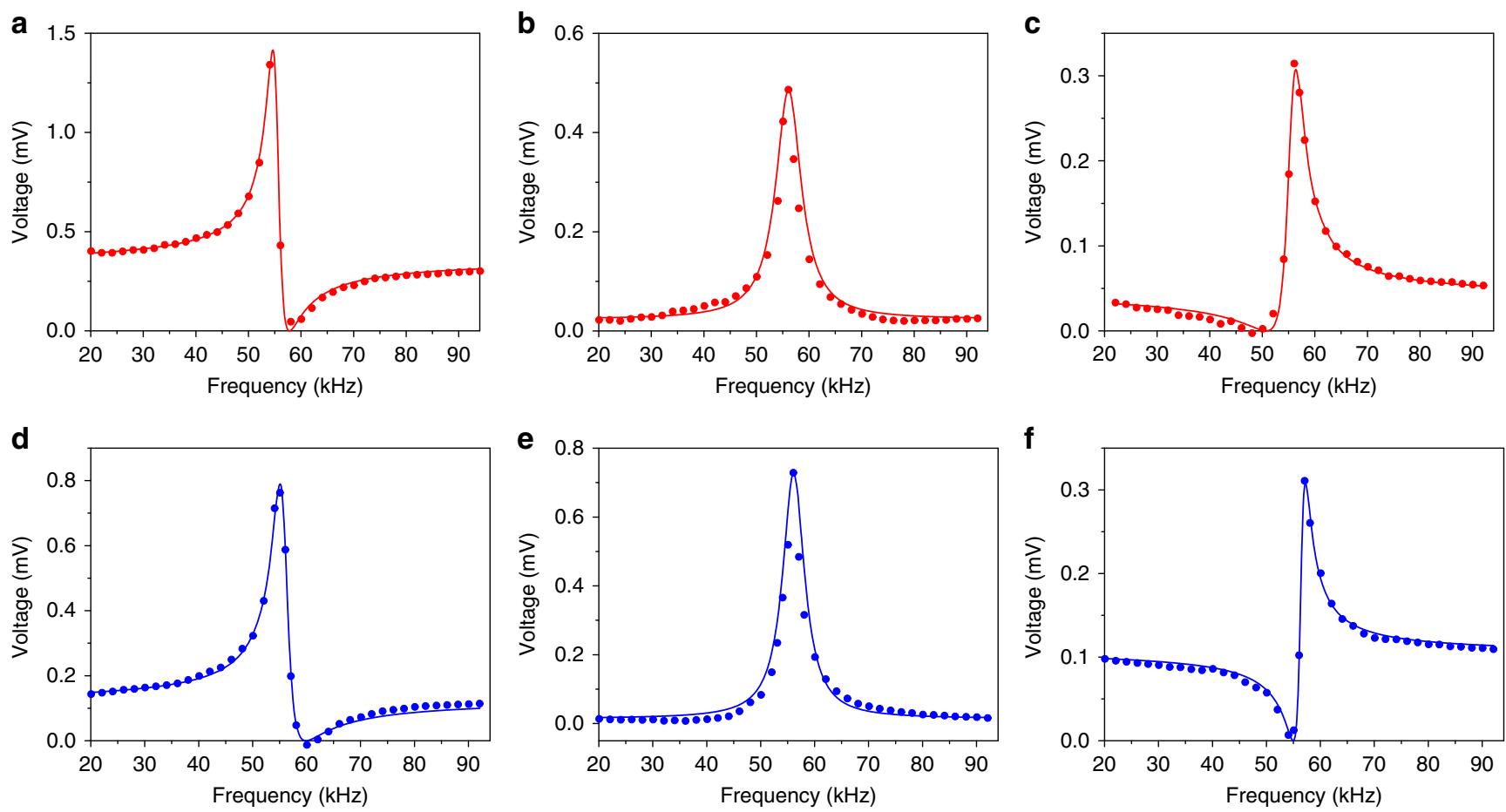

Fig. 3 Representation of anti-resonance effect of $M E$ output voltage. a-c $M E$ output voltage of hot-press sample as a function of $\mathbf{H}_{\mathrm{ac}}$ frequency under $\mathbf{a} \mathbf{H}_{\mathrm{dc}}=4.2 \mathrm{Oe}, \mathbf{b} \mathbf{H}_{\mathrm{dc}}=10.8 \mathrm{Oe}$, and $\mathbf{c} \mathbf{H}_{\mathrm{dc}}=12.8 \mathrm{Oe}$. d-f $\mathrm{ME}$ output voltage of air-dried sample as a function of $\mathbf{H}_{\mathrm{ac}}$ frequency under $\mathbf{d} \mathbf{H}_{\mathrm{dc}}=3.9$ Oe, $\mathbf{e} \mathbf{H}_{\mathrm{dc}}=5.9 \mathrm{Oe}$, and $\mathbf{f} \mathbf{H}_{\mathrm{dc}}=12.7$ Oe. The experimental data (dots) are fitted to a modified Maxwell Eq. 4 or Lorentzian function (shown as solid lines)

The $\alpha_{\mathrm{ME}}$ is also derived from a ME equivalent circuit method $^{25}$, which is expressed as ${ }^{9}$ :

$$
\alpha_{\mathrm{ME}}=\frac{n d_{33, \mathrm{~m}} d_{31, \mathrm{p}}}{n \varepsilon_{0} \varepsilon_{33}^{S} S_{11}^{E}+(1-n) S_{33}^{H}\left(\varepsilon_{0} \varepsilon_{33}^{S}+d_{31, p}^{2} / s_{11}^{E}\right)}
$$

where $n$ is the magnetic phase thickness ratio $s_{11}^{E}$, and $\varepsilon_{33}^{S}$ are the elastic compliances of the piezoelectric and magnetostrictive layers. $\varepsilon_{0}$ and $\varepsilon_{33}^{S}$ are the vacuum permittivity and the dielectric constant of piezoelectric layer at constant strain. $d_{33, \mathrm{~m}}$ and $d_{31, \mathrm{p}}$ are the longitudinal piezomagnetic and transverse piezoelectric coefficients, respectively. According to the measured $\alpha_{\mathrm{ME}}$, elastic moduli (Supplementary Fig. 1) 26 $^{2}$ and dielectric constant ${ }^{27}$ of regenerated cellulose films, and the magnetostrictive coefficient of Metglas SA ${ }^{9}$, the effective $d_{31, \mathrm{p}}$ is estimated as 5.95 and $1.55 \mathrm{pC} \mathrm{N}^{-1}$ for hot-press and air-dried cellulose films, respectively. These values are similar to early reported results of pristine and electrically aligned cellulose films ${ }^{28}$ but is much lower than those of optimized PVDF copolymers (the highest $d_{31}$ can reach up to $28.6 \mathrm{pC} \mathrm{N}^{-1}$ ), and by using this polymer coupling with Metglas SA1, the maximum $\alpha_{\mathrm{ME}}$ of $320 \mathrm{~V} \mathrm{~cm}^{-1} \mathrm{Oe}^{-1}$ has been achieved ${ }^{29}$. Since the Young's modulus are measured as 3.95 $\mathrm{GPa}$ for both the two cellulose films, the ME response should mainly depend on the crystallinity degree of the regenerated cellulose. Thus, it is reasonable to assume that optimization of cellulose matrix could be an effective way to enhance the piezoelectric effect and further improve the $\alpha_{\mathrm{ME}}$ of the cellulosebased ME composite.

Fano resonance of the ME effect. Remarkably, a Fano-resonance effect is observed to accompany the resonance enhancement (Fig. 3). First, the hot press shows a symmetrical Lorentzian resonance profile at an applied $\mathbf{H}_{\mathrm{dc}}=10.8 \mathrm{Oe}$ (Fig. $3 \mathrm{~b}$ ) and as given earlier in Fig. 2a. However, a Fano-like resonance, featuring an anti-resonance peak, appears when the $\mathbf{H}_{\mathrm{dc}}$ is below (Fig. 3a) and above (Fig. 3c) the $\mathbf{H}_{\mathrm{dc}}$ of 10.8 Oe. At a $\mathbf{H}_{\mathrm{dc}}<10.8 \mathrm{Oe}$, the anti-resonance peak occurs at a frequency value that is higher than the resonant peak (Fig. 3a), while if $\mathbf{H}_{\mathrm{dc}}>10.8 \mathrm{Oe}$, the antiresonance damping peak occurs at lower frequencies (Fig. 3c). Similarly, the air-dried sample shows a Fano-like resonance, with a shift in the anti-resonance occurring at $\mathbf{H}_{\mathrm{dc}}$ above and below the $\mathbf{H}_{\mathrm{dc}}(5.9 \mathrm{Oe})$ for the symmetrical Lorentzian resonance (Fig. 3d-f).

To assess the Fano-resonance profile, the $\alpha_{\mathrm{ME}}$ output voltage curves measured as a function of $\mathbf{H}_{\mathrm{dc}}$ are fitted to a modified Maxwell equation:

$$
V(\omega)=A\left|\frac{\omega^{2}-2 i \delta_{\mathrm{a}} \omega_{\mathrm{a}} \omega-\omega_{\mathrm{a}}^{2}}{\omega^{2}-2 i \delta_{\mathrm{r}} \omega_{\mathrm{r}} \omega-\omega_{\mathrm{r}}^{2}}\right|+a \omega+b
$$

where $A$ is the amplitude constant. $\omega_{\mathrm{r}}=2 \pi f_{\mathrm{r}}$ is the resonance frequency and $\omega_{\mathrm{a}}=2 \pi f_{\mathrm{a}}$ is the anti-resonance frequency. $\delta_{\mathrm{r}}$ and $\delta_{\mathrm{a}}$ are the damping constants for the resonance and anti-resonance, respectively. $a$ is the constant corresponding to a linear background voltage and $b$ is the factor fitting the imaginary section to experimental data. We subtracted the background noise and only present the actual ME output voltage here. Fits to the data (solid line) are given in Fig. 3 and all fitting parameters are summarized for the hot-pressed and air-dried samples in Supplementary Tables 1 and 2 .

Fano resonances are widely observed in different fields of physics, e.g., photonic materials and crystals ${ }^{30}$, and recently reviewed for plasmonic nanoparticles and metamaterials ${ }^{31}$. They are also seen in electromechanical coupling of piezoelectric materials, e.g., in impedance measurements of common lead zirconate titanate $\mathrm{s}^{32}$ and generally described as occurring due the interference between continuum and discrete states. However, the Fano resonances of piezoelectric materials have shown not to impose on the frequency line shape of $\mathrm{ME}$ responses $^{32}$ and 

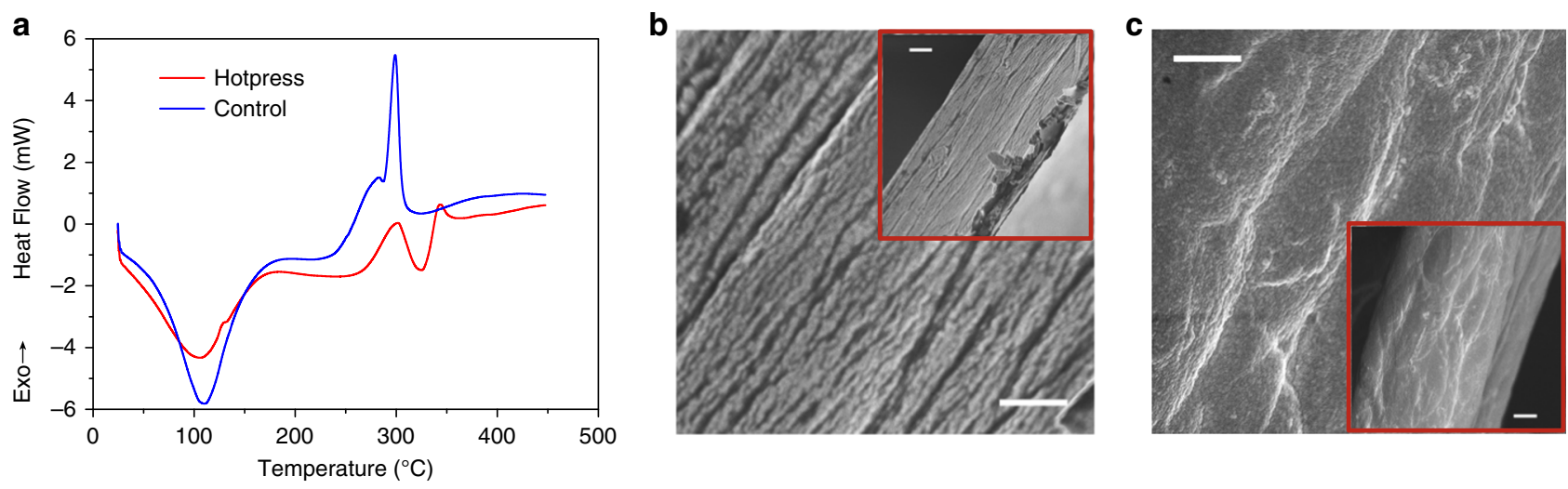

Fig. 4 The effect of treatment on cellulose crystallinity and morphology. a DSC thermograms of hot-press (red line) and control (blue line) films. The melting and oxidation temperatures are 302.9 and $345.5^{\circ} \mathrm{C}$ for hot-press film; 281.3 and $299.0^{\circ} \mathrm{C}$ for air-dried film. The endothermic peak in this range corresponds to melting process of cellulose ${ }^{42}$. The difference of oxidation (decomposition) temperature of the two films has been verified by using TGA (Supplementary Fig. 5). b, c SEM cross-section images of $\mathbf{b}$ hot-pressed and $\mathbf{c}$ air-dried cellulose films. The magnification is $\times 15,000$ for detailed view (scale bar, $1 \mu \mathrm{m}$ ) and $\times 5,000$ for full view (inset, scale bar, $2 \mu \mathrm{m}$ )

therefore until now have not yet been experimentally observed in ME composites, including both polymer (i.e., PVDF) and surprisingly ceramic-based composites that were first established more than four decades ago. Theoretical modeling has predicted Fano resonances either as a conductivity change ${ }^{33}$ or the interference of two excitation pathways of ME laminate cantilever structures ${ }^{34}$, or those oscillating with free ends $s^{35}$. Though despite these models, the lack of experimental evidence suggests that cellulose as a piezoelectric material within ME composites possess unique piezoelectric mechanisms, mechanical properties, and coupling that contribute to the occurrence of a Fano resonance.

To further understand the origin of the Fano resonance, the possible role that water may play is investigated by assessing cellulose with varying degrees of water content. Compared to the ME frequency response of cellulose films prepared at room temperature and with hot pressing under $60^{\circ} \mathrm{C}$ (Figs. 2 and 3), cellulose films hot pressed at higher temperatures of $100^{\circ} \mathrm{C}$ to remove most of the residual water also shows a Fano-resonance effect with the frequency peak of the symmetric Lorentz profile occurring at significantly higher $\mathbf{H}_{\mathrm{dc}}$ field (Supplementary Fig. 2). More specifically, the resonance peak shifts from 5.9 Oe in airdried sample and 10.8 Oe in the $60^{\circ} \mathrm{C}$ hot-pressed samples up to 13.1 Oe in the $100^{\circ} \mathrm{C}$ hot-pressed sample (Supplementary Fig. 3a). Shifts in the frequency values of the anti-resonance, either positioned above or below the resonance peak, also occur due to variations in the water content (Supplementary Fig. 3b) and are qualitatively related to changes in the quality factor, i.e., damping of the ME frequency response. That is, with less water content the resonance and anti-resonance peaks become broader (Supplementary Fig. 2, hot pressed $100^{\circ} \mathrm{C}$ ) as opposed to being narrower if the water content is increased (Fig. $3 \mathrm{a}-\mathrm{c}$, hot pressed $60{ }^{\circ} \mathrm{C}$ ). A further increase in water content gives the narrowest resonance peak, or highest-quality factor, in the air-dried films (Fig. 3a-c, air dried). These changes in the frequency profile indicate that water has a significant effect on the ME response, including the Fano resonance, which still persists when removal of most water is expected. In an attempt to completely eliminate the effects of water and to produce highly homogenous crystalline cellulose, without the amorphous regions found in regenerated cellulose, we prepare ME laminate composites based on nanocrystalline cellulose to further elucidate the Fano resonance. In this case, the highly crystalline form has almost no porous structure, thus minimizing the effect of residual water and heterogeneity (i.e., crystalline vs. amorphous regions) in the piezoelectric layer. From these ME composites using the same configuration and dimensions, a Fano resonance is present although the anti-resonance only occurs at frequencies below the resonance and extraordinarily no symmetrical resonance profile is observed at any applied $\mathbf{H}_{\mathrm{dc}}$ field (Supplementary Fig. 4). Collectively, water plays an ostensible role in the ME response; however, experiments using nanocrystalline cellulose suggest that it is the unique and inherent chemical structure of cellulose that gives rise to the Fano-resonance effect.

At the bulk scale, the strain coupling of the piezoelectric and magnetostrictive can be divided into two pathways: the tension strain caused by magnetostriction and bending strain that is attributed to the configurational asymmetry of a bilayer, shrinkage of epoxy while drying, and mechanical properties of the cellulose. When the tension and bending strains are at equilibrium, the resonance frequency curve of the $\alpha_{\mathrm{ME}}$ output voltage is a symmetrical Lorentzian profile. However, at $\mathbf{H}_{\mathrm{dc}}$ values less or greater than $\mathbf{H}_{\mathrm{dc}}=$ equilibrium, where the driving force for tension strain is either too weak or strong, then interference coupling on the equilibrium bending-tension strain results in a Fano resonance profile.

The equilibrium strain coupling giving a symmetrical Lorentzian profile is observed at $\mathbf{H}_{\mathrm{dc}}=10.8 \mathrm{Oe}$ for the hot pressed (Fig. 2a) and $\mathbf{H}_{\mathrm{dc}}=5.9$ Oe for air dried (Fig. 2b). Since equivalent procedures and magnetostrictive components are used to fabricate the laminates, a difference in the anti-resonance dependence on $\mathbf{H}_{\mathrm{dc}}$, as well as the damping coefficients (Supplementary Tables 1 and 2), from the fitting are likely to be attributed to the different frequency-dependent strain transfer and tensile capacity of the cellulose as a consequence of their different processing methods (i.e., air dried vs. hot pressed). Further to this, anti-resonance is useful for deconvolving the properties of complex mechanically coupled systems, and will be fundamentally important for elucidating the ME mechanisms in cellulose.

Crystallinity and morphology of cellulose. The overall increased performance of hot-press cellulose ME laminate compared to the air dried (Fig. 2d) is presumably due to the piezoelectric properties of each cellulose film, as determined by the extent of crystallinity. To verify this, differential scanning calorimetry (DSC) is employed to study hot-pressed and air-dried cellulose films by quantifying the heat associated with melting of the polymer (Fig. 4a). Thermal analysis of the endothermic peaks 

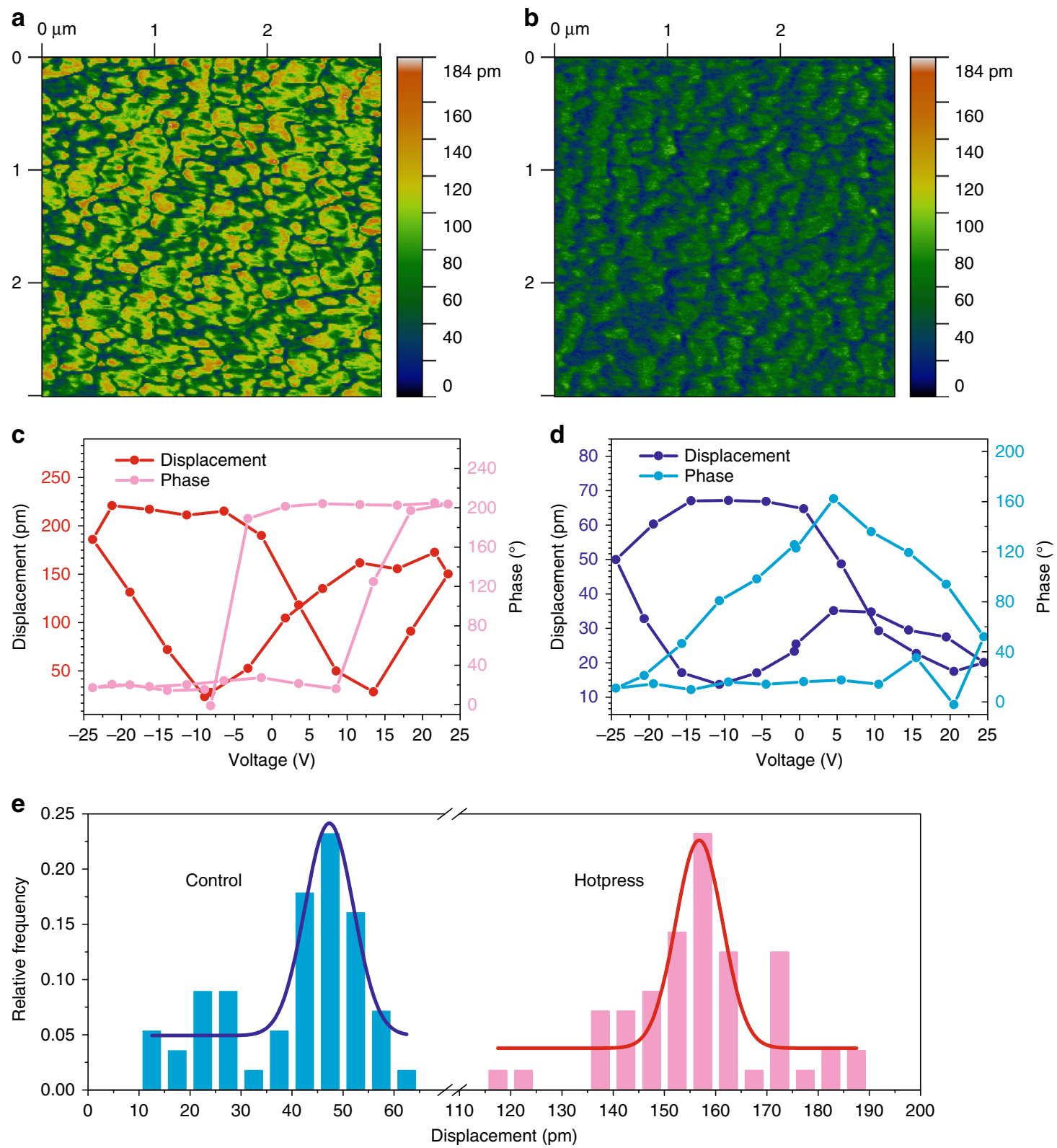

Fig. 5 Effect of treatment on piezoelectric response measured by PFM. a, b PFM amplitude images of a hot-press and $\mathbf{b}$ air-dried samples. A conductive tip is used to apply a constant bias of $9.4 \mathrm{~V}$ to induce local ME displacement while imaging. $\mathbf{c}$, $\mathbf{d}$ Piezoelectric butterfly loops of $\mathbf{c}$ hot-press and $\mathbf{d}$ control cellulose films elucidated by using SS-PFM. The red and blue dots are the hysteresis loops representing the bias-induce amplitude displacement. The pink and cyan dots represent phase changes corresponding to the hysteresis loops. e Histograms of bias induced amplitude displacement at applied voltage of $-25 \mathrm{~V}$ during SS-PFM measurements

corresponding to the melting process indicates the hot-pressed sample adsorbs significantly higher energy, with fusion enthalpies of $50.49 \mathrm{~J} \mathrm{~g}^{-1}$ and $9.39 \mathrm{Jg}^{-1}$ for the hot pressed and air dried, respectively, suggesting a higher crystalline content in former. Further, thermogravimetric analyses indicate that the two different cellulose decompose at different temperature (Supplementary Fig. 4). The difference of crystalline content is explained by changes in cellulose morphology, as evident in scanning electron microscopy (SEM) images of film cross-sections (Fig. 4b, c). In hot-pressed films, the cellulose displays a layered, or aligned fibrous structure, consisting of smaller fibrils with uniform distribution along the longitudinal direction of the film (Fig. 4b). In contrast, the air dried shows a less distinct anisotropic structure, particularly with the absence of smaller fibril structures (Fig. 4c).
Piezoelectric properties of cellulose by piezoresponse force microscopy. Piezoresponse force microscopy (PFM) is used to understand the relationship between the cellulose crystallinity, residual water content, ME output voltage, and the local piezoelectric properties of the hot-press vs. air-dried films. Corresponding PFM height images reveal topography does not significantly differ (Supplementary Fig. 6), while the spatial distribution of the piezoelectric nanoscale domains are similar for the hot-pressed vs. air-dried samples (Fig. 5a, b). Conversely, the phase (Supplementary Fig. 6) and amplitude signal (Fig. 5a, b) indicate that the magnitude of the piezoelectric response within domains is significantly different. For hot-pressed films, the amplitude response within domains is in excess of $100 \mathrm{pm}$ (yellow-to-orange regions), with surrounding regions having values of $\sim 60 \mathrm{pm}$ (darker green) (Fig. 5a). The amplitude 
response from air dried is significantly lower with values of $\sim 60 \mathrm{pm}$ within domains (darker green) and surrounding regions $<40 \mathrm{pm}$ (blue) (Fig. 5b). Domain sizes of $\sim 100-200 \mathrm{~nm}$ are interpreted as piezoresponsive regions rather than single crystalline domains, and previous PFM studies show similar domain sizes in biological tissues ${ }^{36}$. In addition, Supplementary Fig. 6 shows the morphology and phase images directly corresponding to the amplitude images in Fig. 5. The piezoresponsive regions (brighter regions) in Fig. 5a, b are of similar size but do not clearly correlate to the position of globular structures of the surface topography, confirming there is no cross talk in the PFM measurements.

PFM switching spectroscopy (SS-PFM) measurements are further employed to elucidate the polarization switching dynamics of the differently treated cellulose films. Representative hysteresis loops of the hot-pressed sample (Fig. 5c) appear as a typical butterfly shape in the amplitude displacement (red curve) and the corresponding phase change is $\approx 180^{\circ}$, confirming a fully reversible polarization dynamic ${ }^{37,38}$. The air-dried film, on the other hand, shows an unsaturated piezoresponse at positive biases (Fig. 5d), as indicated by significantly less amplitude displacement located on the right wing of the butterfly loop compared to the left wing or negative bias. In addition, the corresponding phase changes are far below $180^{\circ}$, indicating that a completely reversible polarization process is not achieved. This lower amplitude displacement and incomplete polarization switching is attributed to the lower-crystallinity degree though the shielding effect of residual water could also be a contributing factor in aired dried films. Generally, there are two types of water molecules in regenerated cellulose film, namely, the free water and bound water in the cellulose matrix ${ }^{19}$. Free water molecules are easily removed by heating treatment, however, the bound water can persist even at high temperatures. Statistical analysis of biasinduced piezoresponse at a maximum of $-25 \mathrm{~V}$ shows the histogram peak distribution of the amplitude displacement for the hot-pressed film under $60^{\circ} \mathrm{C}$ is threefold higher than the air dried (Fig. 5e), thus confirming differences observed in the PFM amplitude images. Similarly, the hot-pressed films under $100^{\circ} \mathrm{C}$ show significantly higher amplitude displacement in the butterfly curves (Supplementary Fig. 7a), with peak distribution values of $\sim 160 \mathrm{pm}$ (Supplementary Fig. $7 \mathrm{~b}$ ) that are comparable to the $60{ }^{\circ} \mathrm{C}$ films. Therefore, a decrease in the water content accompanied by an increase in crystallinity gives rise to higher piezoelectric response and complete polarization in hot-pressed films.

\section{Discussion}

In conclusion, we fabricate $\mathrm{ME}$ composites using cellulose as the active piezoelectric material, leading to considerable $\alpha_{\mathrm{ME}}$ coefficients of $1.41 \mathrm{~V} \mathrm{~cm}^{-1} \mathrm{Oe}^{-1}$. The cellulose and improvement of its crystallinity, piezoelectric properties, and consequently the ME output is easily achieved using simple and inexpensive solution processing methods. A Fano-like resonance, consisting of an anti-resonance dependence on the magnetic field strength, appears to be due to the unique chemical structure and properties of the cellulose. The asymmetric Fano-resonance profile demonstrates that it is possible to shift the ME output voltage from a peak value to zero sharply, enabling for accurate control of a relatively broad range of power output by easily manipulating the applied magnetic field, and further studies on the resonance line shape will be fundamental for understanding cellulose-based $\mathrm{ME}$ composites. The $\mathrm{ME}$ composites also exploit the current demand for cellulose as a renewable and cheap material, as well as biocompatible and biodegradable properties $^{39}$ that, e.g., will progress the development of $\mathrm{ME}$ composites in a range of applications. In doing so, the study successfully demonstrates the concept of using naturally occurring piezoelectric biopolymers though we anticipate that other piezoelectric proteins and biological materials (e.g., collagen) will importantly find their way into ME composites.

\section{Methods}

Cellulose films preparation. Cellulose solution was prepared by using anhydrous dimethylacetamide (DMAc, Sigma-Aldrich 271012)/LiCl (Sigma-Aldrich 746460) solvent system and headed to $80{ }^{\circ} \mathrm{C}$ with constant stirring. The w.t. \% of cellulose, DAMc, and $\mathrm{LiCl}$ are 1, 9, and $90 \%$, respectively. To increase the solubility, $\alpha$-cellulose (Sigma-Aldrich C8002) was first processed with pre-solution exchange method $^{40}$. Briefly, the cellulose powder was suspended in DI water over night, and then moved to methanol for $1 \mathrm{~h}$. After filtration, the cellulose was exchanged alternately in methanol and DMAc for four times. The result mixture of cellulose and anhydrous DMAc was stored under nitrogen protection before use. Cellulose films were fabricated through regular film casting process. Generally, $1.6 \mathrm{~g}$ of cellulose solution was spread on microscopy glass slide and the pre-film solution was evaporated in fume hood overnight. The casted gels were washed by a blended solution of DI water/isopropanol (3:1 in volume) and then immersed in DI water for 3 days with frequent water changing to remove the residual solvent. The cleaned gel-like films were dried in room temperature as control film, or clamped with hexamethyldisilazane-treated hydrophobic glass in vacuum oven at desired temperature to obtain hot-press film.

ME laminate composite fabrication. The cellulose films were sputtering coated with $50 \mathrm{~nm}$ gold layers on both sides as interface electrodes and then were tailored into a $40 \times 8 \mathrm{~mm}$ rectangular by using scalpel. To fabricate the ME laminate composites, a $36 \times 6 \mathrm{~mm}$ Metglas 2605 SA1 plate was glued on the central part of cellulose films using commercial Devcon epoxy.

Bulk ME effect measurement. An alternatingly generated Helmholtz coil was used to apply ac fields from 20.1 to $92.1 \mathrm{KHz}$ and an electromagnet was use to conduct dc field with various strength. Both the ac and dc fields were provided along the length direction of the ME laminate composites. The induced output voltage was recorded by using a model SR8 10 DSP lock-in amplifier.

Local piezoelectricity measurements. The local piezoelectric response of the cellulose films was analyzed by using an asylum MFP-3D atomic force microscopy system and the conductive tip was nanosensors EMF-50 (Pt/Ir coating) with resonant frequency of $68 \mathrm{kHz}$ and spring constant of $2.8 \mathrm{~N} \mathrm{~m}^{-1}$. The morphology, amplitude, and phase images were obtained in dual ac response tracking ${ }^{41}$ with contact resonant frequency around $320 \mathrm{kHz}$. Piezoelectric response was measured as the first-harmonic of bias-induced tip deflection: $d=d_{0}+A \cos (\omega t+\varphi)$, where $d_{0}$ is the equilibrium position of the tip; $A$ is the amplitude and $\omega$ is the frequency of applied bias; $\varphi$ is the phase-yielded information on the polarization direction below the tip. To study the polarization-switching dynamic, the switching spectroscopy technique was used to obtain the local piezoelectric hysteresis loop. For each laminate sample, spectroscopy measurements were acquired across a $3 \times 3 \mu \mathrm{m}^{2}$ area by applying bias in a matrix of $7 \times 8$ points. The voltage during the SS-PFM measurement was applied in the range of $\pm 25 \mathrm{~V}$, with the frequency of $1 \mathrm{~Hz}$, and this loading is expected to switch the polarization component back and forth. We modified the system by connecting an external amplifier because the upper limit of output signal is $\pm 10 \mathrm{~V}$, which is too weak to induce a saturated switching dynamic. The driving amplitude has been settled on $200 \mathrm{mV}$ for all aforementioned measurements.

DSC and TGA measurements. The thermograms of hot press and control cellulose films were obtained by using a TA Instrument Q100 DSC in the range of $25-450{ }^{\circ} \mathrm{C}$ with a heating rate of $10^{\circ} \mathrm{C} \mathrm{min}-1$. TA Instrument SDT Q600 thermal analysis system was employed for thermogravimetric analyses. The measurements were run under an atmosphere with 9:1 oxygen/nitrogen flows from $25-425^{\circ} \mathrm{C}$ at a ramping rate of $10^{\circ} \mathrm{C} \mathrm{min}^{-1}$.

SEM. The cross-section morphology of air-dried and hot-pressed cellulose films were imaged by using SEM (JEOL JSM-7500FA) with the accelerating voltage of $5.0 \mathrm{kV}$ and the emission current of $10 \mathrm{~mA}$. The samples were sticked on a SEM specimen holder for cross-section imaging and sputter coated with $50 \mathrm{~nm}$ thick gold layer.

Data availability. Data are available from authors on reasonable request 
Received: 29 March 2016 Accepted: 2 May 2017

Published online: 28 June 2017

\section{References}

1 Landau, L. D. et al. Electrodynamics of Continuous Media. Vol. 8 (Elsevier, 1984).

2 Kimura, T. et al. Magnetic control of ferroelectric polarization. Nature 426 55-58 (2003).

3 Spaldin, N. A. \& Fiebig, M. The renaissance of magnetoelectric multiferroics. Science 309, 391-392 (2005).

4 Eerenstein, W., Mathur, N. D. \& Scott, J. F. Multiferroic and magnetoelectric materials. Nature 442, 759-765 (2006)

$5 \mathrm{Ma}$, J., Hu, J., Li, Z. \& Nan, C.-W. Recent progress in multiferroic magnetoelectric composites: from bulk to thin films. Adv. Mater. 23, 1062-1087 (2011).

6 Wang, Y. et al. An extremely low equivalent magnetic noise magnetoelectric sensor. Adv. Mater. 23, 4111-4114 (2011).

7 Suchtelen, J. V. Product properties: a new application of composite materials. Philips Res. Rep 27, 28-37 (1972).

8 Silva, M. et al. Optimization of the magnetoelectric response of poly(vinylidene fluoride)/epoxy/vitrovac laminates. ACS Appl. Mater. Interfaces 5, 10912-10919 (2013).

9 Zhai, J., Dong, S., Xing, Z., Li, J. \& Viehland, D. Giant magnetoelectric effect in metglas/polyvinylidene-fluoride laminates. Appl. Phys. Lett. 89, 083507 (2006).

10 Dong, S., Zhai, J., Li, J. \& Viehland, D. Near-ideal magnetoelectricity in highpermeability magnetostrictive/piezofiber laminates with a (2-1) connectivity. Appl. Phys. Lett. 89, 252904 (2006).

11 Martins, P. \& Lanceros-Méndez, S. Polymer-based magnetoelectric materials. Adv. Funct. Mater. 23, 3371-3385 (2013).

12 Lee, B. Y. et al. Virus-based piezoelectric energy generation. Nat. Nanotechnol. 7, 351-356 (2012)

13 Nguyen, V., Zhu, R., Jenkins, K. \& Yang, R. Self-assembly of diphenylalanine peptide with controlled polarization for power generation. Nat. Commun. 7, 13566 (2016).

14 Jarvis, M. Chemistry: cellulose stacks up. Nature 426, 611-612 (2003).

15 Fukada, E. Piezoelectricity of wood. J. Phys. Soc. Jpn 10, 149-154 (1955).

16 Csoka, L. et al. Piezoelectric effect of cellulose nanocrystals thin films. ACS Macro Lett. 1, 867-870 (2012).

17 Nogi, M. \& Yano, H. Transparent nanocomposites based on cellulose produced by bacteria offer potential innovation in the electronics device industry. $A d v$. Mater. 20, 1849-1852 (2008).

$18 \mathrm{Zhu}$, H. et al. Biodegradable transparent substrates for flexible organic-lightemitting diodes. Energy Environ. Sci. 6, 2105-2111 (2013).

$19 \mathrm{Kim}$, J., Yun, S. \& Ounaies, Z. Discovery of cellulose as a smart material. Macromolecules 39, 4202-4206 (2006).

20 Kim, J., Yun, K., Kim, J. \& Kim, J. Mechanical stretching effect on the actuator performance of cellulose electroactive paper. Smart Mater. Struct. 18, 055005 (2009).

21 Srinivasan, G. Magnetoelectric composites. Annu. Rev. Mater. Res. 40, 153-178 (2010).

22 Zhai, J., Xing, Z., Dong, S., Li, J. \& Viehland, D. Magnetoelectric laminate composites: an overview. J. Am. Ceram. Soc. 91, 351-358 (2008).

$23 \mathrm{Shi}$, Z. et al. Magnetoelectric sensor with miniature universal tunable bias magnetic circuit. Appl. Phys. Lett. 103, 032903 (2013).

24 Gonçalves, R. et al. Synthesis of highly magnetostrictive nanostructures and their application in a polymer-based magnetoelectric sensing device. Eur. Polym. J. 84, 685-692 (2016).

25 Shuxiang, D., Jie-Fang, L. \& Viehland, D. Longitudinal and transverse magnetoelectric voltage coefficients of magnetostrictive/piezoelectric laminate composite: theory. IEEE Trans. Ultrason. Ferroelect. Freq. Control 50, 1253-1261 (2003).

26 Yun, S., Chen, Y., Nayak, J. N. \& Kim, J. Effect of solvent mixture on properties and performance of electro-active paper made with regenerated cellulose. Sens. Actuators B Chem. 129, 652-658 (2008).

27 Yun, G., Kim, J. \& Kim, J. Dielectric and polarization behaviour of cellulose electro-active paper (EAPap). J. Phys. D Appl. Phys. 42, 082003 (2009).

28 Yun, S., Jang, S., Yun, G. \& Kim, J. Electrically aligned cellulose film for electro-active paper and its piezoelectricity. Smart Mater. Struct. 18, 117001 (2009).

29 Jin, J. et al. Multiferroic polymer laminate composites exhibiting high magnetoelectric response induced by hydrogen-bonding interactions. $A d v$. Funct. Mater. 24, 1067-1073 (2014).

30 Fan, S. \& Joannopoulos, J. D. Analysis of guided resonances in photonic crystal slabs. Phys. Rev. B 65, 235112 (2002).
31 Luk'yanchuk, B. et al. The Fano resonance in plasmonic nanostructures and metamaterials. Nat. Mater. 9, 707-715 (2010).

32 Bichurin, M. I. et al. Resonance magnetoelectric effects in layered magnetostrictive-piezoelectric composites. Phys. Rev. B 68, 132408 (2003).

33 Nguyen, T. T., Mininger, X., Bouillault, F. \& Daniel, L. Finite element harmonic modeling of magnetoelectric effect. IEEE Trans. Magn. 47, 1142-1145 (2011).

34 Gerken, M. Resonance line shape, strain and electric potential distributions of composite magnetoelectric sensors. AIP Adv. 3, 062115 (2013).

35 Hasanyan, D. et al. Theoretical and experimental investigation of magnetoelectric effect for bending-tension coupled modes in magnetostrictivepiezoelectric layered composites. J. Appl. Phys. 112, 013908 (2012).

36 Liu, Y., Zhang, Y., Chow, M.-J., Chen, Q. N. \& Li, J. Biological ferroelectricity uncovered in aortic walls by piezoresponse force microscopy. Phys. Rev. Lett. 108, 078103 (2012).

$37 \mathrm{Li}$, Y. et al. Magnetoelectric quasi-(0-3) nanocomposite heterostructures. Nat. Commun. 6, 6680 (2015).

38 Polking, M. J. et al. Ferroelectric order in individual nanometre-scale crystals. Nat. Mater. 11, 700-709 (2012).

39 Klemm, D., Heublein, B., Fink, H.-P. \& Bohn, A. Cellulose: fascinating biopolymer and sustainable raw material. Angew. Chem. Int. Ed. 44, 3358-3393 (2005).

40 McCormick, C. L., Callais, P. A. \& Hutchinson, B. H. Solution studies of cellulose in lithium chloride and N,N-dimethylacetamide. Macromolecules 18 2394-2401 (1985).

41 Brian, J. R., Clint, C., Sergei, V. K. \& Roger, P. Dual-frequency resonancetracking atomic force microscopy. Nanotechnology 18, 475504 (2007).

42 Pedersoli Júnior, J. L. Effect of cellulose crystallinity on the progress of thermal oxidative degradation of paper. J. Appl. Polym. Sci. 78, 61-66 (2000).

\section{Acknowledgements}

The authors acknowledge the financial support from the ARC Australian Research Fellowship (M.J.H.) and ARC DP110104359 and ARC Centre of Excellence for Electromaterials Science (ACES, Project Number CE 140100012), University of Wollongong (UOW). We acknowledge assistance from Yi Du and Long Ren (UOW) for SEM. The authors acknowledge the FCT-Fundação para a Ciência e Tecnologia-for financial support under project PTDC/EEI-SII/5582/2014 and FCT Grant SFRH/BPD/ 96227/2013 (P.M.). S.L.-M. thanks financial support from the Basque Government Industry Department under the ELKARTEK Program and the financial support from the Spanish Ministry of Economy and Competitiveness (MINECO) through the project MAT2016-76039-C4-3-R (AEI/FEDER, UE).

\section{Author contributions}

Y.Z. conducted all experiments and data analysis. T.Z. and P.M. assisted with the ME set-up and bulk measurements. P.M., S.L.-M., Z.Y. and M.J.H. assisted with data analysis and interpretation. Y.Z. and M.J.H. designed the experiments and wrote the manuscript with comments from all authors. Z.Y. and M.J.H. supervised the project.

\section{Additional information}

Supplementary Information accompanies this paper at doi:10.1038/s41467-017-00034-4.

Competing interests: The authors declare no competing financial interests.

Reprints and permission information is available online at http://npg.nature.com/ reprintsandpermissions/

Publisher's note: Springer Nature remains neutral with regard to jurisdictional claims in published maps and institutional affiliations.

Open Access This article is licensed under a Creative Commons Attribution 4.0 International License, which permits use, sharing, adaptation, distribution and reproduction in any medium or format, as long as you give appropriate credit to the original author(s) and the source, provide a link to the Creative Commons license, and indicate if changes were made. The images or other third party material in this article are included in the article's Creative Commons license, unless indicated otherwise in a credit line to the material. If material is not included in the article's Creative Commons license and your intended use is not permitted by statutory regulation or exceeds the permitted use, you will need to obtain permission directly from the copyright holder. To view a copy of this license, visit http://creativecommons.org/ licenses/by/4.0/.

(C) The Author(s) 2017 